\title{
A Novel Extension Method for Transformer Fault Diagnosis
}

\author{
Mang-Hui Wang, Member, IEEE
}

\begin{abstract}
Dissolved gas analysis (DGA) is one of the most useful techniques to detect incipient faults in power transformers. However, the identification of the faulted location by the traditional method is not always an easy task due to the variability of gas data and operational variables. In this paper, a novel extension method is presented for fault diagnosis of power transformers, which is based on the matter-element model and extended relation functions. Thus, incipient faults in power transformers can be directly identified by the degree of relation. The application of this new method to some transformers has yielded promising results.
\end{abstract}

Index Terms-Dissolved gas analysis (DGA), extension theory, matter-element model, transformer fault diagnosis.

\section{INTRODUCTION}

$\mathbf{P}$ OWER TRANSFORMERS are essential devices in a transmission and distribution system. Failure of a power transformer may cause a break in power supply and loss of profits. Therefore, it is of great importance to detect incipient failures in power transformers as early as possible, so that we can switch them safely and improve the reliability of power systems.

A long in-service transformer is subject to electrical and thermal stresses, which may form byproduct gases to indicate the type of incipient failure. Dissolved gas analysis (DGA) is a common practice in the incipient fault diagnosis of power transformers [1], [2]. It tests and periodically samples the insulation oil of transformers to obtain the constituent gases in the oil, which are formed due to breakdown of the insulating materials inside. As study results indicate, corona, overheating, and arcing are the three main causes for insulation degradation in a transformer [2]-[4]. The energy dissipation is the least in corona, medium in overheating, and highest in arcing. The fault-related gases include hydrogen $\left(\mathrm{H}_{2}\right)$, methane $\left(\mathrm{CH}_{4}\right)$, acetylene $\left(\mathrm{C}_{2} \mathrm{H}_{2}\right)$, ethane $\left(\mathrm{C}_{2} \mathrm{H}_{6}\right)$, carbon monoxide $(\mathrm{CO})$, and carbon dioxide $\left(\mathrm{CO}_{2}\right)$.

In the past, various fault diagnosis techniques have been proposed, including the conventional key gas method, ratio method [2], [5], and recently, the expert systems [6], [7], neural-network (NN) [8], and fuzzy logic approaches [9]-[11]. The conventional key gas or ratio method is based on experience in fault diagnosis using DGA data, which may vary from utility to utility due to the heuristic nature of the method and no general mathematical formulation can be used. The expert system and fuzzy logic approaches can take human expertise,

Manuscript received October 22, 2001; revised April 22, 2002. This work was supported in part by the National Science Council, Taipei, Taiwan, R.O.C.

The author is with the Department of Electrical Engineering, National Chin-Yi Institute of Technology, Taiping, Taichung, 411 Taiwan, R.O.C. (e-mail: wangmh@chinyi.ncit.edu.tw).

Digital Object Identifier 10.1109/TPWRD.2002.803838 and have been successfully applied in this field. However, there are some intrinsic shortcomings, such as the difficulty of acquiring knowledge and maintaining a database. The NNs can directly acquire experience from the training data, and exhibit highly nonlinear input-output relationships. This can overcome some of the shortcomings of the expert system. However, the training data must be sufficient and compatible to ensure proper training. A further limitation of the $\mathrm{NN}$ approach is the inability to produce linguistic output. Recently, the combinations of fuzzy logic and AI approach have yielded promising results in the field [6], [12]-[15].

In this paper, a novel extension method is presented for fault diagnosis in power transformers. Cai [16] originally created the concept of extension theory to solve contradictions and incompatibility problems in 1983. In this world, there are some problems that cannot be directly solved by given conditions, but the problem may become easier or solvable through some proper transformation. For example, the Laplace transformation is one of the commonly used techniques in engineering fields and the concept of fuzzy sets is a generalization of well-known standard sets to extend application fields. Therefore, the concept of an extension set is to extend the fuzzy logic value from $[0,1]$ to $(-\infty, \infty)$, which allows us to define any data in the domain and has yielded promising results in many fields [16]-[19]. This paper is the first application of extension theory on power-transformer diagnosis. The proposed extension diagnosis method is based on a matter-element model and an extended set. Thus, the incipient faults of power transformers can be directly identified by the degrees of extended relational functions. Results from applications to some transformers show that the proposed method is fast and more suitable as a practical solution.

\section{REVIEW OF EXTENSION THEORY}

In the standard set, an element either belongs to or does not belong to a set, so the range of the standard set is $\{0,1\}$, which can be used to solve a two-valued problem. In contrast to the standard set, the fuzzy set enables the description of concepts where the boundary is not explicit. It concerns not only whether an element belongs to the set but also to what degree it belongs to. The range of a fuzzy set is $[0,1]$. The extension set extends the fuzzy set from $[0,1]$ to $(-\infty, \infty)$. As a result, it allows us to define a set that includes any data in the domain. Extension theory tries to solve the incompatibility or contradiction problems by the transformation of the matter element. The comparisons of the standard sets, fuzzy sets, and extension sets are shown in Table I. Some definitions of extension theory are introduced in the next section. 
TABLE I

THREe DifFERENT Sorts of MathEMATICAL SETS

\begin{tabular}{l|l|l|l}
\hline $\begin{array}{l}\text { Compared } \\
\text { item }\end{array}$ & Standard set & Fuzzy set & Extension set \\
\hline $\begin{array}{l}\text { Research } \\
\text { objects }\end{array}$ & Data variables & $\begin{array}{l}\text { Linguistic } \\
\text { variables }\end{array}$ & $\begin{array}{l}\text { Contradictory } \\
\text { problems }\end{array}$ \\
\hline Model & $\begin{array}{l}\text { Mathernatics } \\
\text { model }\end{array}$ & $\begin{array}{l}\text { Fuzzy } \\
\text { mathematics } \\
\text { model }\end{array}$ & $\begin{array}{l}\text { Matter-element } \\
\text { model }\end{array}$ \\
\hline $\begin{array}{l}\text { Descriptive } \\
\text { function }\end{array}$ & $\begin{array}{l}\text { Transfer } \\
\text { function }\end{array}$ & $\begin{array}{l}\text { Membership } \\
\text { function }\end{array}$ & Correlation function \\
$\begin{array}{l}\text { Descriptive } \\
\text { property }\end{array}$ & Precision & Ambiguity & Extension \\
$\begin{array}{l}\text { Range of } \\
\text { set }\end{array}$ & $C_{A}(x) \in(0, I)$ & $\mu_{A}(x) \in[0, I]$ & $K_{A}(x) \in(-\infty, \infty)$ \\
\hline
\end{tabular}

\section{A. Matter-Element Theory}

1) Definition of Matter Element: Defining the name of a matter by $N$, one of the characteristics of the matter by $c$, and the value of $c$ by $v$, a matter element in extension theory can be described as follows [16], [19]:

$$
R=(N, c, v)
$$

where $N, c$ and $v$ are called the three fundamental elements of the matter element. For example, $R=$ (John, weight, $80 \mathrm{~kg}$ ) can be used to state that John's weight is $80 \mathrm{~kg}$.

2) Multidimensional Matter Element: Assuming $R=(N$, $C, V)$ is a multidimensional matter element, $C=\left[c_{1}, c_{2}\right.$, $\left.\ldots, c_{n}\right]$ is a characteristic vector, and $V=\left[v_{1}, v_{2}, \ldots, v_{n}\right]$ is a value vector of $C$, then a multidimensional matter element is defined as

$$
R=\left[\begin{array}{c}
N, c_{1}, v_{1} \\
c_{2}, v_{2} \\
\ldots \cdots \\
c_{n}, v_{n}
\end{array}\right]=\left[\begin{array}{c}
R_{1} \\
R_{2} \\
\cdots \\
R_{n}
\end{array}\right]
$$

where $R_{i}=\left(N, c_{i}, v_{i}\right)(i=1,2 \cdots n)$ is defined as the submatter element of $\mathrm{R}$, which can be simplified as follows:

$$
R=(N, C, V) .
$$

3) Divergence of Matter Element: A matter may have many characteristics; the same characteristics and values may also belong to some other matter. In extension theory, there are some formulations to express this as follows:

Theory 1: If a matter has many characteristics, it can be written as

$$
N \dashv(N, v) \dashv\left\{\left(N, c_{1}, v_{1}\right),\left(N, c_{2}, v_{2}\right), \ldots,\left(N, c_{n}, v_{n}\right)\right\} .
$$

The symbol "-f" indicates the mean of the extension.

Theory 2: If some matter has the same characteristic, it can be written as

$$
(N, c, v) \dashv\left\{\left(N_{1}, c, v_{1}\right),\left(N_{2}, c, v_{2}\right), \ldots,\left(N_{n}, c, v_{n}\right)\right\} .
$$

Theory 3: If some matter has the same value, it can be written as

$$
(N, c, v) \dashv\left\{\left(N_{1}, c_{1}, v\right),\left(N_{2}, c_{2}, v\right), \ldots,\left(N_{n}, c_{n}, v\right)\right\} .
$$

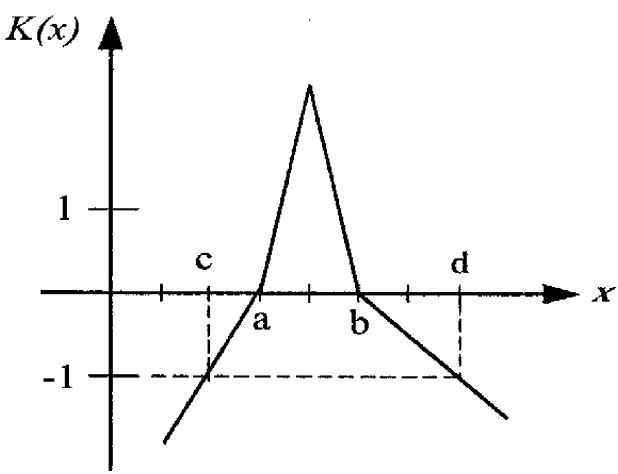

Fig. 1. Extended membership function.

\section{B. Summary of Extension Set Theory}

1) Definition of Extension Set: Let $U$ be a space of objects and $x$ be a generic element of $U$, then an extension set $A$ in $U$ is defined as a set of ordered pairs as follows:

$$
A=\{(u, y) \mid u \in U, y=K(x) \in(-\infty, \infty)\}
$$

where $y=K(x)$ is called the relational function for extension set $A$. The $K(x)$ maps each element of $U$ to a membership grade between $-\infty$ and $\infty$. The higher the degree, the closer the element belongs to the set. Under a special condition, when $0 \leq K(x) \leq 1$, it corresponds to a normal fuzzy set. $K(x) \leq-1$ implies that the element $\mathrm{x}$ has no chance to belong to the set. When $-1<K(x)<0$, it is called an extension domain, which means that the element $x$ still has a chance to become part of the set.

2) Definition of Distance: Let $x$ be a generic point of space $(-\infty, \infty)$ and $X_{o}=\langle a, b\rangle$ a concerned domain which is called a classical domain. Then the left-rightside distance from $x$ to $X_{o}$ is calculated as follows:

$$
\rho\left(x, X_{o}\right)=\left|x-\frac{a+b}{2}\right|-\frac{b-a}{2} .
$$

3) Distance Values: Let $X_{o}=\langle a, b\rangle, X=\langle c, d\rangle$, and $X_{o} \in X$, where $X_{o}$ and $X$ are the classical and neighborhood domains, respectively. Then, the left-rightside distance value from $x$ to $X_{o}$ and $X$ is calculated as follows:

$$
D\left(x, X_{o}, X\right)= \begin{cases}\rho(x, X)-\rho\left(x, X_{o}\right) & x \notin X_{o} \\ -1 & x \in X_{o} .\end{cases}
$$

4) Primitively Extended Relation Function: Let $X_{o}=$ $\langle a, b\rangle, X=\langle c, d\rangle$ and $X_{o} \in X$, the extended relation function can be defined as follows:

$$
K(x)=\frac{\rho\left(x, X_{o}\right)}{D\left(x, X_{o}, X\right)} .
$$

The relation function can be used to calculate the membership grade between $x$ and $X_{o}$. The extended membership function is shown in Fig. 1. When $K(x) \geq 0$, it indicates and describes the degrees to which $x$ belongs to $X_{o}$. When $K(x)<0$ it describes the degree to which $x$ does not belong to $X_{o}$. 
TABLE II

IEC GAS RATIO CODES

\begin{tabular}{c|c|c|c}
\hline Ranges of & \multicolumn{3}{|c}{ Codes of different gas ratios } \\
\cline { 2 - 4 } the gas ratio & $\frac{\mathrm{C}_{2} \mathrm{H}_{2}}{\mathrm{C}_{2} \mathrm{H}_{4}}$ & $\frac{\mathrm{CH}_{4}}{\mathrm{H}_{2}}$ & $\frac{\mathrm{C}_{2} \mathrm{H}_{4}}{\mathrm{C}_{2} \mathrm{H}_{6}}$ \\
\hline$<0.1$ & 0 & 1 & 0 \\
\hline $0.1-1$ & 1 & 0 & 0 \\
\hline $1-3$ & 1 & 2 & 1 \\
\hline$>3$ & 2 & 2 & 2 \\
\hline
\end{tabular}

TABLE III

Fault Types AcCording to the Gas Ratio Codes

\begin{tabular}{c|c|c|c|c}
\hline $\begin{array}{l}\text { Fault } \\
\text { type no. }\end{array}$ & Fault type & $\frac{\mathrm{C}_{2} \mathrm{H}_{2}}{\mathrm{C}_{2} \mathrm{H}_{4}}$ & $\frac{\mathrm{CH}_{4}}{\mathrm{H}_{2}}$ & $\frac{\mathrm{C}_{2} \mathrm{H}_{4}}{\mathrm{C}_{2} \mathrm{H}_{6}}$ \\
\hline 1 & No fault & 0 & 0 & 0 \\
\hline 2 & $<150^{\circ} \mathrm{C}$ Thermal fault & 0 & 0 & 1 \\
\hline 3 & $\begin{array}{c}150^{\circ} \mathrm{C} \sim 300{ }^{\circ} \mathrm{C} \\
\text { Thermal fault }\end{array}$ & 0 & 2 & 0 \\
\hline 4 & $\begin{array}{c}300^{\circ} \mathrm{C} \sim 700{ }^{\circ} \mathrm{C} \\
\text { Thermal fault }\end{array}$ & 0 & 2 & 1 \\
\hline 5 & $\begin{array}{c}>700^{\circ} \mathrm{C} \text { Thermal } \\
\text { fault }\end{array}$ & 0 & 2 & 2 \\
\hline 6 & $\begin{array}{c}\text { Low energy partial } \\
\text { discharges }\end{array}$ & 0 & 1 & 0 \\
\hline 8 & $\begin{array}{c}\text { High energy partial } \\
\text { discharges }\end{array}$ & 1 & 1 & 0 \\
\hline 9 & Low energy discharges & 1 or 2 & 0 & 1 or 2 \\
\hline
\end{tabular}

\section{Proposed Extension Diagnosis Method}

In DGA, IEC codes have been used widely by the utilities. For IEC Std. 599, the codes of different gas ratios and fault classifications according to the gas ratio codes are shown in Tables II and III, respectively. Although IEC codes are useful for fault diagnosis in transformers, the number of code combinations is larger than that of fault types. Therefore, "no match" may be indicated in the fault diagnosis. In this section, the extended fault diagnosis method is proposed for power transformers. First, we need to develop matter-element models of the fault types, and then incipient faults of tested transformers can directly be identified by the degree of an extended relation function.

\section{A. Matter-Element Model of Fault Diagnosis}

The first step of the extended fault diagnosis method is to formulate matter-element models of fault types. For IEC Std. 599 [3], there are nine fault types according to the gas ratio codes. In Table III, $R_{i}$ is the matter element of nine fault types, where $I=\left\{I_{1}, I_{2}, I_{3}, I_{4}, I_{5}, I_{6}, I_{7}, I_{8}, I_{9}\right\}$ is the fault set, $I_{i}$ is an $i$ th fault type, $C=\left\{C_{1}, C_{2}, C_{3}\right\}$ is a characteristic set in which
TABLE IV

Fault MatTer Elements of Dissolved GaS

\begin{tabular}{c|c|c|c}
\hline $\begin{array}{c}\text { Fault } \\
\text { no. }\end{array}$ & 1 & 2 & 3 \\
\hline $\begin{array}{c}\text { Matter- } \\
\text { element }\end{array}$ & $R_{1}=\left\{\begin{array}{r}I_{1}, \mathrm{C}_{1},\langle 0,0.1\rangle \\
\mathrm{C}_{2},\langle 0.1,1\rangle \\
\mathrm{C}_{3},\langle 0,1\rangle\end{array}\right]$ & $R_{2}=\left\{\begin{array}{r}I_{2}, \mathrm{C}_{1},\langle 0,0.1\rangle \\
\mathrm{C}_{2},\langle 0.1,1\rangle \\
\mathrm{C}_{3},\langle 1,3\rangle\end{array}\right]$ & $R_{3}=\left\{\begin{array}{r}I_{1}, \mathrm{C}_{1},\langle 0,0.1) \\
\mathrm{C}_{2},\langle 1,3\rangle \\
\mathrm{C}_{3},\langle 0,1\rangle\end{array}\right\}$ \\
\hline $\begin{array}{c}\text { Fault } \\
\text { no. }\end{array}$ & 4 & 5 & 6 \\
\hline $\begin{array}{c}\text { Matter- } \\
\text { element }\end{array}$ & $R_{4}=\left\{\begin{array}{c}I_{4}, \mathrm{C}_{1},\langle 0,0.1\rangle \\
\mathrm{C}_{2},\langle 1,3\rangle \\
\mathrm{C}_{3},\langle 1,3\rangle\end{array}\right\}$ & $R_{5}=\left\{\begin{array}{r}I_{5}, \mathrm{C}_{1},\langle 0,0.1\rangle \\
\mathrm{C}_{2},\langle 1,3\rangle \\
\mathrm{C}_{3},\langle 3,10\rangle\end{array}\right]$ & $R_{6}=\left\{\begin{array}{r}I_{6}, \mathrm{C}_{1},\langle 0,0.1\rangle \\
\mathrm{C}_{2},(0,0.1) \\
\mathrm{C}_{3},\langle 0,1\rangle\end{array}\right\}$ \\
\hline $\begin{array}{c}\text { Fault } \\
\text { no. }\end{array}$ & 7 & 9 \\
\hline $\begin{array}{c}\text { Matter- } \\
\text { element }\end{array}$ & $R_{7}=\left\{\begin{array}{c}I_{7}, \mathrm{C}_{1},\langle 0.1,3\rangle \\
\mathrm{C}_{2},\langle 0,0.1\rangle \\
\mathrm{C}_{3},\langle 0,1\rangle\end{array}\right\}$ & $R_{8}=\left\{\begin{array}{r}I_{8}, \mathrm{C}_{1},\langle 0.1,3\rangle \\
\mathrm{C}_{2},\langle 0.1,1\rangle \\
\mathrm{C}_{3},\langle 1,3\rangle\end{array}\right\}$ & $R_{9}=\left\{\begin{array}{r}I_{9}, \mathrm{C}_{1},\langle 0.1,3) \\
\mathrm{C}_{2},\langle 0.1,1\rangle \\
\mathrm{C}_{3},\langle 3,10\rangle\end{array}\right\}$ \\
\hline
\end{tabular}

$C_{1} \rightarrow \mathrm{C}_{2} \mathrm{H}_{2} / \mathrm{C}_{2} \mathrm{H}_{4}, C_{2} \rightarrow \mathrm{CH}_{4} / \mathrm{H}_{2}, C_{3} \rightarrow \mathrm{C}_{2} \mathrm{H}_{2} / \mathrm{C}_{2} \mathrm{H}_{6}$. The value range, classical domains of every characteristic set are according to Tables II and III. The neighborhood domain defined as the possible range of every gas ratio is set as

$$
R_{p}=\left(P, C, V_{p}\right)=\left\{\begin{array}{c}
P, C_{1},\langle 0,10\rangle \\
C_{2},\langle 0,10\rangle \\
C_{3},\langle 0,18\rangle
\end{array}\right\} .
$$

After the element-matter model of fault diagnosis is formulated, the fault diagnosis of power transformers can be initiated.

\section{B. Extension Fault Diagnosis Methods}

The proposed extension diagnosis method has been successfully implemented using PC-based software for fault diagnosis of power transformers. The extension fault diagnosis method is described as follows:

Step 1: Formulating the matter element of every fault type (as in Section III-A) as follows:

$$
R_{i}=\left[\begin{array}{c}
I_{i}, C_{1}, V_{i 1} \\
C_{2}, V_{i 2} \\
C_{3}, V_{i 3}
\end{array}\right] \quad i=1,2,9
$$

where $V_{i j}=\left\langle a_{i j}, b_{i j}\right\rangle$ and $V_{p j}^{\prime}=\left\langle a_{p j}, b_{p j}\right\rangle$ are the classical and neighborhood domains of fault gas ratio, respectively. The ranges of domains can be directly obtained from the IEC-defined range of gas ratios. It also can be determined from previous experience.

Step 2: Formulating the gas matter element (Table IV) of the tested transformer as follows:

$$
R_{t}=\left[\begin{array}{c}
T, C_{1}, v_{t 1} \\
C_{2}, v_{t 2} \\
C_{3}, v_{t 3}
\end{array}\right]
$$




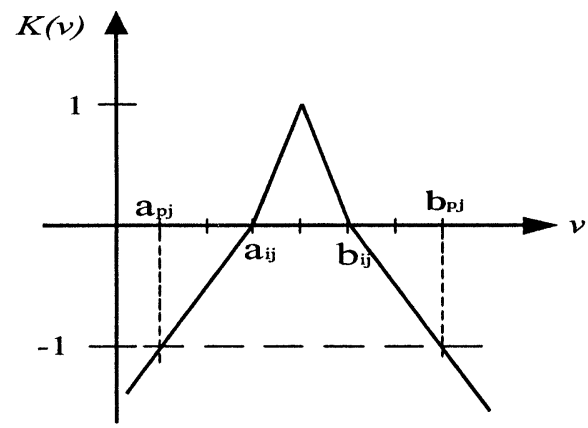

Fig. 2. Proposed extended relation function.

Step 3: Calculating the relation degree of the tested transformer with the faulted characteristic by the proposed extended relation function

$$
\begin{gathered}
K_{i j}\left(v_{t j}\right)= \begin{cases}\frac{-\rho\left(v_{t j}, V_{i j}\right)}{\left|V_{i j}\right|}, & \text { if } v_{t j} \in V_{i j} \\
\frac{\rho\left(v_{t j}, V_{i j}\right)}{\rho\left(v_{t j}, V_{p j}^{\prime}\right)-\rho\left(v_{t j}, V_{i j}\right)}, & \text { if } v_{t j} \notin V_{i j}\end{cases} \\
i=1,2, \ldots, 9 ; j=1,2,3 .
\end{gathered}
$$

The proposed extended relation function can be shown as Fig. 2, where $0 \leq K(v) \leq 1$ corresponds to the normal fuzzy set. It describes the degree to which $v$ belongs to $V$. When $K(v)<0$, it indicates the degree to which $x$ does not belong to $X_{o}$.

Step 4: Setting the weights of the fault pattern, $W_{i 1}, W_{i 2}$, $W_{i 3}$, depends on the importance of every gas ratio in the fault diagnosis process. In this paper, all three of the weights are set at $1 / 3$.

Step 5: Calculating the relation indexes for every fault type.

$$
\lambda_{i}=\sum_{j=1}^{3} W_{i j} K_{i j}, \quad i=1,2, \ldots, 9 .
$$

Step 6: Normalizing the values of the relation indexes into an interval between -2 and 1 as Eq. (16). This process will be beneficial for fault diagnosis.

$$
\lambda_{i}^{\prime}=\frac{3 \lambda-\lambda_{\min }-2 \lambda_{\max }}{\lambda_{\max }-\lambda_{\min }}, \quad i=1,2, \ldots, 9
$$

where

$$
\begin{aligned}
\lambda_{\max } & =\max _{1 \leq i \leq 9}\left\{\lambda_{i}\right\} \\
\lambda_{\min } & =\min _{1 \leq i \leq 9}\left\{\lambda_{i}\right\} .
\end{aligned}
$$

Step 7: Ranking the normalized fault indexes to detect the fault type of the tested transformer. The fault diagnosis rule is shown as follows:

$$
\text { If } \left.\left(\lambda_{k}^{\prime}=1\right) \text { THEN (the fault type is } I_{k}\right) \text {. }
$$

Note that the proposed method can determine the main fault severity compared to other types, and identify the fault likelihood by the fault indexes. It is most helpful in the diagnosis of multiple fault transformers.
TABLE $\mathrm{V}$

Tested Gas Data of Transformers and Diagnosis Results BY DIFFERENT METHOD

\begin{tabular}{c|l|l|l|l|l|c|c|c}
\hline $\begin{array}{r}\text { Tr. } \\
\text { No. }\end{array}$ & $\mathrm{H}_{2}$ & $\mathrm{CH}_{4}$ & $\mathrm{C}_{2} \mathrm{H}_{6}$ & $\mathrm{C}_{2} \mathrm{H}_{4}$ & $\mathrm{C}_{2} \mathrm{H}_{2}$ & $\mathrm{AFN}$ & $\mathrm{EC}$ & $\mathrm{EXM}$ \\
\hline \hline 1 & 14.7 & 3.7 & 10.5 & 2.7 & 0.2 & 1 & 1 & 1 \\
2 & 345 & 112.3 & 27.5 & 51.5 & 58.8 & 8 & 8 & 8 \\
3 & 181 & 262 & 41 & 28 & 0 & 3 & 3 & 3 \\
4 & 173 & 334 & 172 & 812.5 & 37.7 & 5 & 5 & 5 \\
5 & 127 & 107 & 11 & 154 & 224 & 9 & 9 & 9 \\
6 & 60 & 40 & 6.9 & 110 & 70 & 9 & 9 & 9 \\
7 & 220 & 340 & 42 & 480 & 14 & 5 & 5 & 5 \\
8 & 170 & 320 & 53 & 520 & 3.2 & 5 & 5 & 5 \\
9 & 27 & 90 & 42 & 63 & 0.2 & 4 & 4 & 4 \\
10 & 565 & 53 & 34 & 47 & 0 & 8 & $\mathrm{~N}$ & 8 \\
11 & 56 & 286 & 96 & 928 & 7 & 5 & 5 & 5 \\
12 & 200 & 48 & 14 & 117 & 131 & 9 & 9 & 9 \\
13 & 78 & 161 & 86 & 353 & 10 & 5 & 5 & 5 \\
14 & 32.4 & 5.5 & 1.4 & 12.6 & 13.2 & 9 & 9 & 9 \\
15 & 980 & 73 & 58 & 12 & 0 & 6 & 6 & 6 \\
16 & 160 & 130 & 33 & 96 & 0 & 2 & 2 & 2 \\
17 & 650 & 53 & 34 & 20 & 0 & 6 & 6 & 6 \\
18 & 95 & 110 & 160 & 50 & 0 & 3 & 3 & 3 \\
19 & 300 & 490 & 180 & 360 & 95 & 4 & $\mathrm{~N}$ & 4 \\
20 & 200 & 700 & 250 & 740 & 1 & 4,5 & 4 & 4 \\
21 & 625 & 130 & 47 & 2 & 0 & 3 & 3 & 3 \\
22 & 56 & 61 & 75 & 32 & 31 & 7 & $\mathrm{~N}$ & 7 \\
\hline
\end{tabular}

Step 8: Go back to step two for the next transformer when the diagnosis of one has been completed, until all have been done.

The main advantage of the proposed method is that it can provide more detailed information about the fault inside a transformer by relation fault indexes. Moreover, the proposed method does not need to learn or to tune any parameters, and a simple software package can easily implement it.

\section{CASE Studies And Discussions}

To demonstrate the effectiveness of the proposed extension fault diagnosis method, 22 sets of field DGA data from the power transformers in China [8], [9], Australia [10], and Taiwan were tested. The detailed gas data are shown in Table V, where the AFN expresses the actual fault-type number, and the IEC and EXM are the diagnosis results of the IEC method and the proposed extension method, respectively. In Table $\mathrm{V}$, numbers 10,19 , and 22 had no matching codes for diagnosis by the IEC method, but the results of the proposed method showed excellent agreement with actual faults in the transformer. The actual faults were a low-energy discharge, a $300{ }^{\circ} \mathrm{C} \sim 700{ }^{\circ} \mathrm{C}$ thermal fault and high-energy partial discharges, respectively.

The transformer number 20 was diagnosed by the IEC method to have a fault-type number four [i.e., a thermal fault of medium temperature $\left(300 \sim 700{ }^{\circ} \mathrm{C}\right)$ ]. The actual fault of transformer number 20 was that two locations of overheating damage were found due to eddy currents and a bad contact [10]. In comparison, the proposed method indicated that high $\left(>700{ }^{\circ} \mathrm{C}\right)$ and medium temperature $\left(300 \sim 700{ }^{\circ} \mathrm{C}\right)$ faults 
TABLE VI

Relation Degrees $\lambda_{i}^{\prime}$ By the EXTENDED Diagnosis Method (PARTIAL RESULTS)

\begin{tabular}{c||c|c|c|c|c|c|c|c|c||c}
\hline \multicolumn{1}{c||}{$\begin{array}{c}\text { Tr. } \\
\text { no. }\end{array}$} & 1 & 2 & 3 & 4 & 5 & 6 & 7 & 8 & 9 & $\begin{array}{l}\text { Diag- } \\
\text { nosis } \\
\text { results }\end{array}$ \\
\hline 1 & 1.00 & -0.44 & -0.32 & -1.75 & -2.00 & 0.201 & -0.54 & -1.18 & -1.43 & 1 \\
\hline 2 & -0.84 & 0.056 & -1.93 & -1.04 & -2.00 & -1.57 & -0.62 & 1.00 & 0.037 & 8 \\
\hline 3 & 0.11 & -1.12 & 1.00 & -0.23 & -1.11 & -0.37 & -0.37 & -1.12 & -2.00 & 3 \\
\hline 11 & -0.21 & -0.10 & 0.05 & 0.162 & 1.00 & -0.29 & -2.00 & -1.80 & -0.966 & 5 \\
\hline 12 & -0.88 & -0.78 & -2.00 & -1.90 & -1.14 & -1.52 & -0.50 & 0.23 & 1.00 & 9 \\
\hline 13 & -0.65 & -0.40 & 0.31 & 0.56 & 1.00 & -0.82 & -2.00 & -1.57 & -1.13 & 5 \\
\hline 19 & -1.62 & -0.09 & -0.53 & 1.00 & -0.53 & -2.00 & -1.19 & 0.713 & -0.82 & 4 \\
\hline 20 & -0.19 & 0.52 & 0.297 & 1.00 & 0.944 & -0.32 & -2.00 & -1.17 & -1.23 & $4,5^{*}$ \\
\hline 22 & 0.273 & -1.68 & 0.508 & -1.45 & -2.00 & -0.51 & 1.00 & -0.17 & -0.73 & 7 \\
\hline
\end{tabular}

Note: the * means the second likelihood fault type.

existed. The extended fault indexes indicating the likelihood of each fault are 0.944 and 1, respectively, in Table VI. The fuzzy logic method [10] also can diagnose the two faults. These two methods were capable of pointing toward multiple faults, but the proposed method did not need to learn or to tune any parameters, and it could be easily implemented by computer software.

The partial relation degrees of tested transformers are shown in Table VI. It is very easy to diagnose the fault types in power transformers from Table V. For example, in transformer number one, the relation degree with the fault-type number one is equal to one (or maximum value), which is indicative of fault-type number one, or no faults. In comparison, the relation degrees with other fault types are very small. Therefore, transformer number one does not need to be checked in the future. Moreover, the proposed method cannot only diagnose the main fault types of power transformers. It can also provide useful information for future trend analysis by the relation degree. For example, transformer number 13 was diagnosed to have main fault-type number five $\left(>700{ }^{\circ} \mathrm{C}\right.$ thermal fault). On the other hand, the relation degree, approximately 0.56 , also shows that this transformer had a medium possibility of fault-type number four (i.e., $300{ }^{\circ} \mathrm{C} \sim 700{ }^{\circ} \mathrm{C}$ thermal fault). Conversely, because of a negative relation degree, transformer number 13 had a very low possibility of fault-type number seven.

Taiwan Electric Research and Testing Center (TERTC) supplied the data for transformer number 21 and 22 . The information included the transformer number, the sampling data, the amount of TCG, and past remedies of transformer. The proposed method agreed well with the actual fault, where transformer number 21 is an overheat (slight) fault and transformer number 22 is high-energy partial discharges in the bare metal.

To test the diagnosis performance of the proposed method, diagnosis accuracy with different noise percentages is shown in Table VII. The sources of error include measurement, instruments, human mistakes, etc., which could lead to data uncertainties. To take into account the errors and uncertainties, a set of the
TABLE VII

Diagnosis PeRformances OF DifFERENT METHOdS With DiFFERENT PERCENTAGES OF ERRORS ADDED

\begin{tabular}{l|l|l|c}
\hline \multirow{2}{*}{$\begin{array}{l}\text { Noise } \\
\text { Percentage (\%) }\end{array}$} & \multicolumn{2}{|l|}{ Proposed Method } & \multirow{2}{*}{$\begin{array}{c}\text { IEC } \\
\text { Method }\end{array}$} \\
\cline { 2 - 3 } & $\begin{array}{l}\text { Only using 1st } \\
\text { relation degree }\end{array}$ & $\begin{array}{l}\text { Using 1st and 2nd } \\
\text { relation degree }\end{array}$ & \\
\hline $\pm 0 \%$ & $96 \%$ & $100 \%$ & $83 \%$ \\
\hline $\pm 5 \%$ & $91 \%$ & $95 \%$ & $60 \%$ \\
\hline $\pm 10 \%$ & $91 \%$ & $95 \%$ & $48 \%$ \\
\hline $\pm 15 \%$ & $83 \%$ & $95 \%$ & $43 \%$ \\
\hline $\pm 20 \%$ & $74 \%$ & $95 \%$ & $26 \%$ \\
\hline $\pm 25 \%$ & $61 \%$ & $90 \%$ & $17 \%$ \\
\hline $\pm 30 \%$ & $52 \%$ & $81 \%$ & $17 \%$ \\
\hline
\end{tabular}

testing data was created by adding $\pm 5 \%$ to $\pm 30 \%$ random uniform-distributed samples to test the robustness of the proposed method. As shown in Table VII, the proposed method in both cases of relation degree has a significantly higher diagnosis accuracy of $96 \%$ and $100 \%$, respectively. In comparison, the diagnosis accuracy of the IEC method is only $83 \%$ due to a missing one of multifault cases and three no-matching codes. Moreover, the proposed method shows good tolerance to add errors, and has a high accuracy of $81 \%$ in an extreme error of $\pm 30 \%$.

\section{CONCLusions}

This paper presents a novel fault diagnosis method based on the extension theory for power transformers. Compared with other traditional AI methods, the proposed method does not require particular artificial parameters and learning processes. In addition, the calculation of the proposed diagnosis algorithm is fast and very simple. It can be easily implemented by PC software. Test results show that the proposed method cannot only diagnose the main fault types of power transformers; it can also detect useful information for future trends and multifault analysis by the relation degrees. Moreover, the proposed method has a significantly high degree of diagnosis accuracy and shows good tolerances to errors added.

\section{REFERENCES}

[1] J. J. Kelly, "Transformer fault diagnosis by dissolved-gas analysis," IEEE Trans. Ind. Applicat., vol. IA-16, pp. 777-782, Dec. 1980.

[2] R. R. Rogers, "IEEE and IEC codes to interpret faults in transformers, using gas in oil analysis," IEEE Trans. Elect. Insulation, vol. 13, pp. 349-354, Oct. 1978.

[3] "Interpretation of the analysis of gases in transformers and other oilfilled electrical equipment in service," Book, IEC Publication 599, 1st ed., 1978.

[4] IEEE Guide for the Interpretation of Gases Generated in Oil-Immersed Transformers, IEEE Std. C57. 104-1991.

[5] E. Dornenburg and W. Strittmater, "Monitoring oil cooling transformers by gas analysis," Brown Boveri Review, vol. 61, pp. 238-274, 1974.

[6] C. E. Lin, J. M. Ling, and C. L. Huang, "An expert system for transformer fault diagnosis using dissolved gas analysis," IEEE Trans. Power Delivery, vol. 8, pp. 231-238, Jan. 1993.

[7] Y. Zhang, X. Ding, Y. Liu, and P. J. Griffin, "An artificial neural network approach to transformer fault diagnosis," IEEE Trans. Power Delivery, vol. 11, pp. 1836-1841, Oct. 1996.

[8] Z. Wang, Y. Liu, and P. J. Griffin, "A combined ANN and expert system tool for transformer fault diagnosis," IEEE Trans. Power Delivery, vol. 13, pp. 1224-1229, Oct. 1998. 
[9] G. Zhang, K. Yasuoka, and S. Ishii, "Application of fuzzy equivalent matrix for fault diagnosis of oil-immersed insulation," in Proc. 13th Int. Conf. on Dielectric Liquids, Nara, Japan, July 20-25, 1999, pp. 400-403.

[10] Q. Su, C. Mi, L. L. Lai, and P. Austin, "A fuzzy dissolved gas analysis method for the diagnosis of multiple incipient faults in a transformer," IEEE Trans. Power Syst., vol. 15, pp. 593-598, May 2000.

[11] Q. Su, "A fuzzy logic tool for transformer fault diagnosis," in Int. Conf. Power Syst. Technol., 2000 Proc., vol. 1, 2000, pp. 265-268.

[12] J. J. Dukarm, "Transformer oil diagnosis using fuzzy logic and neural networks," in 1993 Canadian Conf. Elect. Comput. Eng., vol. 1, 1993, pp. 329-332.

[13] K. Tomsovic, M. Tapper, and T. T. Ingvarsson, "A fuzzy information approach to integrating different transformer diagnostic methods," IEEE Trans. Power Delivery, vol. 8, pp. 1638-1643, July 1993.

[14] Y. C. Huang, H. T. Yang, and C. L. Huang, "Developing a new transformer diagnosis system through evolutionary fuzzy logic," IEEE Trans. Power Delivery, vol. 12, pp. 761-767, Apr. 1997.

[15] J. J. Dukam, "Transformer oil diagnosis using fuzzy logic and neural networks," in Canadian Conf. Elect. Comput. Eng., vol. 1, 1993, pp. 329-332.

[16] W. Cai, "The extension set and incompatibility problem," J. Scientific Exploration, vol. 1, pp. 81-93, 1983.
[17] - "Extension set, fuzzy set and classical set," in Proc. First Congress Int. Fuzzy Syst. Assoc., Spain, 1985.

[18] J. Li and S. Wang, Primary Research on Extension Control Information System, China: International Academic Publishers, 1991, vol. 1.

[19] Y. P. Huang and H. J. Chen, "The extension-based fuzzy modeling method and its applications," in Proc. IEEE Canadian Conf. Elect. Comput. Eng., Edmonton, AB, Canada, May 1999, pp. 977-982.

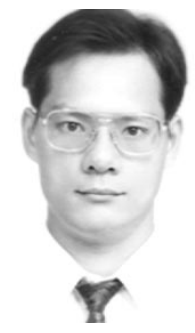

Mang-Hui Wang (S'90-M'94) was born in Taitung, Taiwan, R.O.C., on June 22, 1963. He received the M.Sc. and Ph.D. degrees from the Electrical Engineering Department at National Taiwan University of Science and Technology, Taipei, Taiwan, in 1990 and 1994, respectively.

Currently, he is an Associate Professor in the Electrical Engineering Department at National Chin-Yi Institute of Technology, Taichung, Taiwan, R.O.C., where he has been since August 1994. His major areas of research include power system stability and AI application to power systems. 